\title{
IL SUFFISSO -ZIONE/-CIÓNIN ITALIANO ED IN SPAGNOLO: ANALISI E TRATTAMENTO DEI DATI PER INCLUSIONE NEL DIES/DEIS (DICCIONARIO ITALIANO-ESPAÑOL DE SUFIJOS/DICCIONARIO ESPAÑOL-ITALIANO DE SUFIJOS)
}

\author{
Antonella D'Angelis \\ Universidad de Sevilla
}

\begin{abstract}
Resumen: Este trabajo representa una propuesta para el análisis y el tratamiento del sufijo -zione/-ción en italiano y español y su inclusión en el diccionario de sufijos italiano-español/español-italiano (DIES/DEIS). Partiendo de un estudio pormenorizado de los ejemplos existentes de palabras en -zione/-ción proponemos un modelo de ficha de diccionario que recoja información lingüística en ambas lenguas y sea capaz de proporcionarla de forma clara tanto en soporte electrónico como en soporte papel.
\end{abstract}

Palabras clave: Morfología, sufijos, diccionario, italiano, español.

Abstract: This work is a proposal for analysis and treatment of the suffix -zione/-ción in Italian and Spanish and its inclusion in the ItalianSpanish/Spanish Italian suffix dictionary (DIES/DEIS). Having carried out a comprehensive analysis of existing examples of words in -zione/-ción, we intend to propose a model of suffix dictionary card that contains clear linguistic information in both languages and in electronic and paper format.

Keywords: Morphology, suffixes, dictionary, Italian, Spanish.

\section{INTRODUZIONE}

Questo lavoro si è reso necessario per verificare la validità della struttura della base dati FileMaker Pro ${ }^{1}$ per la realizzazione delle varie fasi del DIES/DEIS di cui ci stiamo occupando negli ultimi anni della nostra ricerca.

Il DIES/DEIS (Diccionario Italiano-Español de Sufijos / Diccionario Español-Italiano de Sufijos) si articolerà in due parti: la prima parte in supporto cartaceo e la seconda in supporto elettronico.

Ciascuna delle due parti avrà uno sviluppo diverso, dal momento che esistono difficoltà considerevoli a riprodurre in supporto elettronico dei riferimenti

${ }^{1}$ FileMaker Pro è un programma per la creazione di base dati che funziona tanto in ambiente Windows che Macintosh. 
incrociati tra i vari suffissi, le equivalenze suffissali a livello semantico dei vari suffissi, le corrispondenze o divergenze suffissali dell'italiano o dello spagnolo per coprire la stessa semantica, ecc.

In un lavoro precedente (d'Angelis/Núñez Román, 2013:203), a proposito dello sviluppo del dizionario in sopporto cartaceo affermavamo:

En su primera parte en soporte papel el DIES/DEIS está pensado como un sistema de fichas de sufijos italiano-español y viceversa, en los que estén presentes informaciones de diferente naturaleza:

- lema: en este apartado aparecerá el sufijo italiano y el sufijo español y viceversa;

- definición: se proporcionarán datos referentes al tipo de palabras que forma cada uno de los sufijos y la categoría gramatical de procedencia, añadiendo además información etimológica de forma resumida;

- extensión cronológica: en este apartado pondremos la época en la que el sufijo empieza a ser usado o deja de serlo;

- campo: entendemos con este término cualquier referencia a una determinada ciencia, técnica o disciplina;

- frecuencia: en este apartado se usará la etiqueta raro, para indicar que en la actualidad es poco frecuente;

- uso: en este apartado se pondrá figurado, extensivo, irónico, jocoso, eufemistico o despectivo, para subrayar que un sufijo produce lemas que se usan de una determinada manera;

- registro: usaremos este campo para marcar un registro familiar, literario, popular, regional, vulgar, etc. Indicará lemas con estas características;

- ejemplos;

- estadística de uso del sufijos a nivel diacrónico (desde su aparición hasta la actualidad) y si produce transcategorización.

Durante la nostra ricerca, abbiamo dovuto constatare, purtroppo, che l'ipotesi teorica iniziale del progetto, che abbiamo appena riportato sopra, non si riflette pienamente nelle necessità reali dell'organizzazione della base dati FileMaker Pro, necessità che emergono immediatamente nel momento stesso in cui si considerano tutti gli esempi delle parole suffissate con lo stesso suffisso e i loro equivalenti spagnoli, e che questi campi devono essere considerati anche nella parte elettronica. Inoltre ciascuno dei campi ipotizzati deve obbligatoriamente essere considerato come un'interazione di singole tabelle relazionali per poter poi successivamente permettere qualsiasi tipo di ricerca incrociata all'interno della base dati.

Abbiamo, pertanto, pensato che per la verifica del modello teorico iniziale, dovessimo considerare il suffisso -zione ed il suo equivalente spagnolo -ción. Si è trattata di una scelta dovuta alla necessità di studiare ed analizzare un suffisso i cui esempi nel dizionario Sabatini Coletti fossero numerosi, poiché a maggior numero di esempi corrisponde comè ovvio un maggior numero di casi divergenti in quanto a eccezioni, restrizioni, suffissi stessi, ecc. 


\section{IL SUFFISSO-ZIONE/-CIÓN IN ITALIANO ED IN SPAGNOLO: ANALISI} E TRATTAMENTO DEI DATI.

A differenza del tipo di analisi realizzata in (d'Angelis/Núñez Román, 2013: 206-212) per il suffisso italiano - ume ed il suo equivalente spagnolo -ez, in cui come si può vedere in un breve frammento della tabella di seguito riportata, il corpus analizzato includeva: tutti gli esempi presenti (che per ovvie ragioni di spazio non possiamo riportare in questa sede), la data a cui l'esempio risaliva, il tipo di base derivazionale dal quale si formava la parola suffissata, il campo, la frequenza, l'uso ed il registro di ogni singolo esempio:

\begin{tabular}{|l|c|c|c|c|c|c|}
\hline \multicolumn{7}{|l|}{ FASE II DIS: Corpus de ejemplos de-UME } \\
\hline EJEMPLOS & DATACIÓN & BASE & CAMPO & FRECUENCIA & USO & REGISTRO \\
\hline Rozzume & XIII & adj. & & OBSOLETO & & \\
\hline Melume & XIV & sust. & $\begin{array}{c}\text { técn. científico } \\
\text { botánico }\end{array}$ & & & \\
\hline Sucidume & XIV & adj. & técn. científico & & & literario \\
\hline Bastardume & XIV & adj. & & bajo uso & despectivo & \\
\hline Biancume & XIV & adj. & & bajo uso & & \\
\hline Fortume & XIV & adj. & & bajo uso & & \\
\hline Fracidume & XIV & adj. & & bajo uso & & \\
\hline Frittume & XIV & adj. & & bajo uso & despectivo & \\
\hline Grossume & XIV & adj. & & bajo uso & & \\
\hline Mollume & XIV & adj. & & bajo uso & & \\
\hline
\end{tabular}

Nel caso specifico del suffisso analizzato -zione e dato il copioso numero di esempi di parole suffissate con questo suffisso, ciò non è stato possibile giacché per il nostro scopo iniziale bastava considerare che il suffisso formasse nomi da verbi, quindi il tipo di base derivazionale era sempre la stessa, la deverbale.

Abbiamo limitato quindi la nostra analisi a tutti gli esempi di parole suffissate in -zione, presenti nel dizionario italiano Sabatini Coletti, con tutte le definizioni presenti ed abbiamo tradotto tutti i singoli significati in spagnolo e da ciò abbiamo potuto verificare che spesso si producevano delle differenze derivative morfologiche tra italiano e spagnolo, come potremmo vedere più avanti.

Abbiamo analizzato pertanto 2488 parole suffissate con tutti i rispettivi significati, i campi, la datazione delle parole, l'etimologia, procedendo quindi alla traduzione in spagnolo come si può vedere di seguito.

Nella tabella seguente possiamo osservare che compare in alto il sUFFISSO BASE con la DEFINIZIONE dello stesso, e di seguito gli ESEMPI DEL SUFFISSO -zione, la DEFINIZIONE di ogni parola suffissata, la TRADUZIONE IN SPAGNOLO e un'ultima colonna, che abbiamo chiamato DIFFERENZE DI USO, in cui si registrano invece gli usi diversi dei suffissi in italiano e spagnolo ed eventualmente si inserisce 
anche l'informazione inerente al suffisso diverso evidenziato in verde ed usato offrendo anche la definizione corrispondente dello stesso. Quando non sono presenti differenze di uso, semplicemente si usa un NON CI sono.

Alla luce dei dati analizzati, ci sembra molto interessante considerare la possibilità di poter mettere in relazione i vari esempi italiani e le corrispondenti traduzioni in spagnolo in modo tale che sia il significato il punto di connessione tra le due lingue, dal momento che in diversi casi non esistono né corrispondenza di base derivativa né equivalenza di suffissi.

Abbiamo ritenuto opportuno in questa sede proporre solamente un paio di esempi estratti dal corpus di suffissati in -zione, dei quasi 2500 esempi analizzati in 750 pagine di dati, considerando che gli esempi proposti sono esemplificativi dell'analisi realizzata in tutti i casi considerati:

\begin{tabular}{|c|c|c|c|}
\hline Suffisso baSE & \multicolumn{3}{|c|}{ DEFINIZIONE } \\
\hline -ZIONE & \multicolumn{3}{|c|}{$\begin{array}{l}\text { SUFFISSO NOMINALE DEVERBALE CHE FORMA SOSTANTIVI, GENERALMENTE, ASTRATTI } \\
\text { INDICANTI L'AZIONE, L'EFFETTO O IL RISULTATO ESPRESSO DAL VERBO. }\end{array}$} \\
\hline $\begin{array}{l}\text { ESEMPI } \\
\text { SUFFISSO } \\
\text {-ZIONE }\end{array}$ & DEFINIZIONE & $\begin{array}{c}\text { TRADUZIONE } \\
\text { SPAGNOLA } \\
\text { ESEMPI }\end{array}$ & $\begin{array}{l}\text { DIFFERENZE } \\
\text { DI USO }\end{array}$ \\
\hline 1. Abbreviazione & $\begin{array}{l}\text { 1. Riduzione a proporzioni o dimensioni } \\
\text { minori [SIN] accorciamento: a. del servizio } \\
\text { di leva } \\
\text { 2. Accorciamento di una parola; la parola } \\
\text { così ridotta: segno di a.; l'elenco delle a. } \\
\text { del dizionario; forma ridotta di una parola } \\
\text { usata in luogo di quella intera: cine è l'a. di } \\
\text { cinematografo; dott. è l'a. di dottore } \\
\text { 3. ling. Riduzione da lunga a breve della } \\
\text { quantità di una vocale } \\
\text { 4. mus. Negli spartiti, annotazione fatta con } \\
\text { parole accorciate o segni convenzionali } \\
\text { 5. dir. a. dei termini, riduzione del tempo } \\
\text { stabilito dalla legge per giungere a un } \\
\text { dibattimento } \\
\text { [ETIM] dal lat. tardo abbreviatiónem, deriv. } \\
\text { di abbreviàre "abbreviare" } \\
\text { a sec. XIV }\end{array}$ & $\begin{array}{l}\text { 1. Abreviación } \\
\text { 2. Abreviatura } \\
\text { (Del Lat. } \\
\text { Abbreviatūra). } \\
\text { 3. Abreviación } \\
\text { 4. Abreviatura }\end{array}$ & $\begin{array}{l}1 \text { e 3: NON CI } \\
\text { SONO } \\
2 \text { e 4: Alternanza - } \\
\text { ZIONE/-TURA. } \\
\text { Il suffisso spagno- } \\
\text { lo -TURA suffisso } \\
\text { nominale deverbale } \\
\text { che forma sostanti- } \\
\text { vi, indicanti l'azione, } \\
\text { l'effetto o il risultato } \\
\text { espresso dal verbo. }\end{array}$ \\
\hline 2. Abdicazione & $\begin{array}{l}\text { 1. Rinuncia al trono } \\
\text { 2. estens. Rinuncia a un diritto, a un dovere, } \\
\text { a una prerogativa, anche in senso fig.: a. al } \\
\text { dovere; a. all'incarico; a. ai propri sentimenti } \\
\text { [ETIM] dal lat. abdicatiōnem, deriv. di } \\
\text { abdicāre "abdicare"; nell'accez. } 1 \text { attrav. il fr. } \\
\text { abdication } \\
\text { a sec. XIV }\end{array}$ & $\begin{array}{l}\text { 1. Abdicación } \\
\text { 2. }\end{array}$ & NON CI SONO \\
\hline
\end{tabular}


3. DIVERGENZE SUFFISALI SPAGNOLE NELL'USO DI-CIÓN RISPETTO A-ZIONE

Proponiamo di seguito delle tabelle con tutte le divergenze suffissali spagnole rispetto allo stesso suffisso italiano -zione, purtroppo per ragioni di spazio non si sono potute includere le informazioni inerenti ai significati delle singole parole analizzate e si è optato per mantenere solo i numeri corrispondenti ai singoli significati. Si marca in neretto la divergenza suffissale e sempre in neretto la divergenza di uso nel caso delle locuzioni.

Ammontano a 199 i casi di parole suffissate con divergenza in spagnolo, come si può osservare di seguito.

\begin{tabular}{|c|c|}
\hline 1. ABBREVIAZIONE & $\begin{array}{l}\text { 1. e 3. ABREVIACIÓN } \\
\text { 2. e 4. ABREVIATURA } \\
\text { 5. non esiste in spagnolo }\end{array}$ \\
\hline 2. ACCOMANDAZIONE & CONTRATO DE VASALLAJE \\
\hline 3. AEROSOSTENTAZIONE & SUSTENTACIÓN AEORODINÁMICA \\
\hline 4. AFFRANCAZIONE & 1.-4. FRANQUEAMIENTO \\
\hline 5. AGGIUNZIONE & AÑADIDURA/ADITAMENTO \\
\hline 6. ALTERAZIONE & $\begin{array}{l}\text { 1-3. ALTERACIÓN } \\
\text { 4. EROSIÓN } \\
\text { 5.- 6. ALTERACIÓN }\end{array}$ \\
\hline 7. ALTERCAZIONE & 1.'e 2. ALTERCADO \\
\hline 8. ANGOLAZIONE & $\begin{array}{l}\text { 1.-3. ANGULACIÓN } \\
\text { 2.ÂNGULO }\end{array}$ \\
\hline 9. APPETIZIONE & APETENCIA \\
\hline 10. ASCOLTAZIONE & $\begin{array}{l}\text { 1. ESCUCHA } \\
\text { 2. AUSCULTACIÓN }\end{array}$ \\
\hline 11. BIGLIETTAZIONE & DISTRIBUCIÓN Y VENTA DE BILLETES Y ENTRADAS \\
\hline 12. BITUMIZZAZIONE & $\begin{array}{l}\text { 1. BITUMINACIÓN } \\
\text { 2. EMBETUNADO }\end{array}$ \\
\hline 13. BONIFICAZIONE & SANEAMIENTO/ABONADO \\
\hline 14. BURRIFICAZIONE & PRODUCCIÓN DE LA MANTEQUILLA \\
\hline 15. CANTILLAZIONE & TARAREAR/CANTURREAR \\
\hline 16. CAPITOLAZIONE & 1. e 2. CAPITULADO \\
\hline 17. CAPRIFICAZIONE & CABAHIGADURA \\
\hline 18. CARCERAZIONE & ENCARCELAMIENTO \\
\hline 19. CARICAZIONE & CARGA \\
\hline 20. CASSAZIONE (2) & $\begin{array}{l}\text { CASSAZIONE } \\
\text { SERENATA }\end{array}$ \\
\hline
\end{tabular}




\begin{tabular}{|c|c|}
\hline 21. CASTIGAZIONE & $\begin{array}{l}\text { 1. CASTIGO } \\
\text { 2. EDMENDACIÓN }\end{array}$ \\
\hline 22. CATRAMAZIONE & ALQUITRANAMIENTO \\
\hline 23. CAVAZIONE & $\begin{array}{l}\text { 1. EXCAVACIÓN } \\
\text { 2. LIBRAR EL HIERRO }\end{array}$ \\
\hline 24. CEDUAZIONE & TALADO \\
\hline 25. CESSAZIONE & CESIÓN \\
\hline 26. COIBENTAZIONE & AISLAMIENTO \\
\hline 27. COLLUTTAZIONE & PELEA \\
\hline 28. COMPARIZIONE & COMPARECENCIA \\
\hline 29. CONFLAGRAZIONE & $\begin{array}{l}\text { 1. CONFLAGRACIÓN } \\
\text { 2. HOSTILIDAD }\end{array}$ \\
\hline 30. CONSULTAZIONE & CONSULTA \\
\hline 31. CONTRORELAZIONE & CONTRAINFORME \\
\hline 32. COSCRIZIONE & RECLUTAMIENTO \\
\hline 33. CRONICIZZAZIONE & CRONICISMO \\
\hline 34. DANNAZIONE & $\begin{array}{l}\text { 1. DAMNACIÓN in disuso per CONDENACIÓN } \\
\text { 2. DESGRACIA } \\
\text { 3. DAMNACIÓN }\end{array}$ \\
\hline 35. DATTILOCOMPOSIZIONE & MECANOGRAFIADO \\
\hline 36. DECANAPULAZIONE & SEPARACIÓN DE LAS FIBRAS DEL YUTE \\
\hline 37. DECAPITAZIONE & $\begin{array}{l}\text { 1. e 2.DECAPITACIÓN } \\
\text { 3. ACORTAMIENTO }\end{array}$ \\
\hline 38. DECIFRAZIONE & DESCIFRE \\
\hline 39. DECONTRAZIONE & $\begin{array}{l}\text { 1. DISTENSIÓN } \\
\text { 2. RELAJACIÓN }\end{array}$ \\
\hline 40. DECORAZIONE & $\begin{array}{l}\text { 1. DECORADO } \\
\text { 2. DECORACIÓN } \\
\text { 3. CONDECORACIÓN }\end{array}$ \\
\hline 41. DECORTICAZIONE & $\begin{array}{l}\text { 1. DESCORTEZO/DESCORTEZAMIENTO } \\
\text { 2. DESOLLADURA } \\
\text { 3. DESCORTEZAMIENTO }\end{array}$ \\
\hline 42. DECOZIONE & $\begin{array}{l}\text { 1. DECOCCIÓN } \\
\text { 2. SUSPENSIÓN DE PAGOS }\end{array}$ \\
\hline 43. DEFALCAZIONE & 1. e 2. DESFALCO. \\
\hline 44. DEGNAZIONE & CONDESCENDENCIA \\
\hline 45. DELUCIDAZIONE (2) & DESLUCIDO \\
\hline 46. DESTINAZIONE & 1. - 3. DESTINO \\
\hline 47. DETASSAZIONE & $\begin{array}{l}\text { DETASACIÓN } \\
\text { REDUCCIÓN DE IMPUETOS }\end{array}$ \\
\hline
\end{tabular}




\begin{tabular}{|c|c|}
\hline 48. DETENZIONE & $\begin{array}{l}\text { 1. e 2.TENENCIA } \\
\text { 3. DETENCIÓN }\end{array}$ \\
\hline 49. DIRAMAZIONE & $\begin{array}{l}\text { 1. -3. RAMIFICACIÓN } \\
\text { 4. DIFUSIÓN }\end{array}$ \\
\hline 50. DISASSUEFAZIONE & DESENGANCHE \\
\hline 51. DISCETTAZIONE & $\begin{array}{l}\text { 1. DISCUSIÓN } \\
\text { 2. ARGUMENTACIÓN }\end{array}$ \\
\hline 52. DISFAZIONE & 1. e 2. DESHECHO \\
\hline 53. DISLOCAZIONE & $\begin{array}{l}\text { 1. - 3. DESPLAZAMIENTO } \\
\text { 4. e 5. DISLOCACIÓN }\end{array}$ \\
\hline 54. DISOLEAZIONE & 1. DESACEITADO \\
\hline 55. DISPUTAZIONE & 1. e 2. DISPUTACIÓN (in disuso)/ DISPUTA/DEBATE \\
\hline 56. DIVARICAZIONE & $\begin{array}{l}\text { 1. ENSANCHAMIENTO } \\
\text { 2. DISCREPANCIA }\end{array}$ \\
\hline 57. DOLORAZIONE & $\begin{array}{l}\text { 1. DOLORIMIENTO } \\
\text { 2. TORMENTO }\end{array}$ \\
\hline 58. DUBITAZIONE & DUBITACIÓN/DUDA \\
\hline 59. EFFUMAZIONE & EXHALACIÓN DE VAPORES \\
\hline 60. ELENCAZIONE & LISTADO \\
\hline 61. ELETTROTRAZIONE & TRACCIÓN ELÉCTRICA \\
\hline 62. EMENDAZIONE & EMENDACIÓN/ENMIENDA \\
\hline 63. ERUTTAZIONE & ERUCTACIÓN/ERUCTO \\
\hline 64. ESPLETAZIONE & CUMPLIMIENTO \\
\hline 65. ESTRANIAZIONE & 1. e 2. EXTRAÑAMIENTO. \\
\hline 66. FARNETICAZIONE & DESVARÍO \\
\hline 67. FERTIRRIGAZIONE & REGADÍO FERTILIZANTE \\
\hline 68. FORMATTAZIONE & FORMATEADO \\
\hline 69. FOTOCONDUZIONE & FOTOCONDUCTIVIDAD \\
\hline 70. FRANCESIZZAZIONE & AFRANCESAMIENTO \\
\hline 71. FUCILAZIONE & FUSILAMIENTO \\
\hline 72. GIRAZIONE & GIRO \\
\hline 73. GIUBILAZIONE & $\begin{array}{l}\text { 1. JÚBILO } \\
\text { 2. JUBILACIÓN }\end{array}$ \\
\hline 74. GIUNZIONE & $\begin{array}{l}\text { 1. JUNTURA } \\
\text { 2. e 3. CONEXIÓN } \\
\text { 4. UNIÓN } \\
\end{array}$ \\
\hline 75. GRASSAZIONE & ATRACO \\
\hline 76. IMBALSAMAZIONE & 1. e 2. EMBALSAMAMIENTO \\
\hline 77. IMMURAZIONE & EMPAREDAMIENTO \\
\hline
\end{tabular}




\begin{tabular}{|c|c|}
\hline 78. IMPOLVERAZIONE & $\begin{array}{l}\text { SULFATADO } \\
\text { SULFATACIÓN }\end{array}$ \\
\hline 79. IMPOSTAZIONE (1) & $\begin{array}{l}\text { 1. CIMENTACIÓN } \\
\text { 2. FORMULACIÓN } \\
\text { 3. PLANTEAMIENTO } \\
\text { 4. e 5. IMPOSTACIÓN } \\
\text { 6. ASENTAMIENTO } \\
\text { 7. IMPOSICIÓN }\end{array}$ \\
\hline 80. IMPOSTAZIONE (2) & DESPACHO DE CARTAS \\
\hline 81. INCANTAGIONE & ENCANTAMIENTO \\
\hline 82. INCHIOSTRAZIONE & ENTINTADO \\
\hline 83. INGROSSAZIONE & ENGROSAMIENTO \\
\hline 84. INTESTAZIONE & 1. e 2. ENCABEZAMIENTO \\
\hline 85. INVENTARIAZIONE & INVENTARIADO \\
\hline 86. IRREGGIMENTAZIONE & ALISTAMIENTO \\
\hline 87. IRRORAZIONE & $\begin{array}{l}\text { 1. ROCIAMIENTO } \\
\text { 2. IRRRIGACIÓN }\end{array}$ \\
\hline 88. LATTAZIONE & LACTANCIA \\
\hline 89. MACELLAZIONE & MATANZA \\
\hline 90. MACINAZIONE & MOLEDURA/MOLIENDA \\
\hline 91. MAGGIORAZIONE & AUMENTO \\
\hline 92. MALEDUCAZIONE & 1.e 2. MALA EDUCACIÓN \\
\hline 93. MATTAZIONE & MATANZA \\
\hline 94. MENOMAZIONE & $\begin{array}{l}\text { 1. MINUSVALÍA } \\
\text { 2. DETERIORO }\end{array}$ \\
\hline 95. MESCOLAZIONE & MEZCLAMIENTO/ MEZCLADURA \\
\hline 96. MISCELAZIONE & MEZCLA \\
\hline 97. MONACAZIONE & TOMA DE HÁBITO \\
\hline 98. MONTICAZIONE & MONTANERA \\
\hline 99. MUTUAZIONE & $\begin{array}{l}\text { 1. INTERCAMBIO } \\
\text { 2. CONVALIDACIÓN }\end{array}$ \\
\hline 100. NEGLEZIONE & NEGLIGENCIA \\
\hline 101. NEOFORMAZIONE & $\begin{array}{l}\text { 1. NEOLOGISMO/NUEVA FORMACIÓN } \\
\text { 2. NEOPLASIA } \\
\text { 3. NUEVA FORMACIÓN }\end{array}$ \\
\hline 102. NOBILITAZIONE & 1. -3. ENNOBLECIMIENTO \\
\hline 103. OSCURAZIONE & OSCURECIMIENTO \\
\hline 104. OSSIDORIDUZIONE & ÓXIDO REDUCCIÓN \\
\hline 105. PALPEBRAZIONE & PARPADEO \\
\hline
\end{tabular}




\begin{tabular}{|c|c|}
\hline 106. PASTURAZIONE & CEBA \\
\hline 107. PATTUIZIONE & РАCTO \\
\hline 108. PERLUSTRAZIONE & RECONOCIMIENTO \\
\hline 109. PERQUISIZIONE & PESQUISA \\
\hline 110. PIANURIZZAZIONE & ALLANAMIENTO \\
\hline 111. PICCHETTAZIONE & ESTACADA/VALLADO \\
\hline 112. PLUVIRRIGAZIONE & RIEGO PLUVIAL \\
\hline 113. POSTAZIONE & 1. e 2. EMPLAZAMIENTO \\
\hline 114. POSTFAZIONE & EPÍLOGO \\
\hline 115. POSTICIPAZIONE & APLAZAMIENTO \\
\hline 116. PRECINZIONE & DIAZOMA \\
\hline 117. PREFAZIONE & PREFACIO \\
\hline 118. PRESSOINIEZIONE & ESTAMPADO DE INYECCIÓN \\
\hline 119. PROPAGOLAZIONE & MULTIPLICACIÓN POR PROPÁGULOS \\
\hline 120. PROSPETTAZIONE & PLANTEAMIENTO \\
\hline 121. QUASSAZIONE & MAJADO \\
\hline 122. RADARLOCALIZZAZIONE & LOCALIZACIÓN POR RADAR \\
\hline 123. RADARNAVIGAZIONE & NAVEGACIÓN POR RADAR \\
\hline 124. RADIOSCINTILLAZIONE & BÚSQUEDA SUBMARINA \\
\hline 125. RADIOSTAZIONE & RADIO EMISORA \\
\hline 126. RAREFAZIONE & ENRARECIMIENTO \\
\hline 127. RASTREMAZIONE & AHUSAMIENTO \\
\hline 128. RATEALIZZAZIONE & PLAZO \\
\hline 129. RATEIZZAZIONE & PLAZO \\
\hline 130. REIEZIONE & RECHAZO/REYECCIÓN \\
\hline 131. RENDICONTAZIONE & RENDICIÓN DE CUENTAS \\
\hline 132. RICETTAZIONE (2) & RECETARIO \\
\hline 133. RICEZIONE & $\begin{array}{l}\text { 1. e 3. e 4. RECEPCIÓN } \\
\text { 2. RECIBIMIENTO } \\
\text { 5. RECIBO/RECIBIMIENTO }\end{array}$ \\
\hline 134. RICOGNIZIONE & 1. - 3. RECONOCIMIENTO \\
\hline 135. RICONGIUNZIONE & CONVERGENCIA \\
\hline 136. RICONVOCAZIONE & VUELTA A CONVOCAR \\
\hline
\end{tabular}




\begin{tabular}{|c|c|}
\hline 137. RICORREZIONE & NUEVA CORRECCIÓN \\
\hline 138. RICREAZIONE & $\begin{array}{l}\text { 1. RECREO } \\
\text { 2. RECREACIÓN }\end{array}$ \\
\hline 139. RIDENOMINAZIONE & $\begin{array}{l}\text { 1. CAMBIO DE NOMBRE } \\
\text { 2. RECONVERSIÓN }\end{array}$ \\
\hline 140. RIESPOSIZIONE & 1. e 2. NUEVA EXPOSICIÓN \\
\hline 141. RIFERMENTAZIONE & SEGUNDA FERMENTACIÓN \\
\hline 142. RIFINIZIONE & 1. e 2. ACABADO \\
\hline 143. RISERVAZIONE & 1. e 2. RESERVA \\
\hline 144. RITARDAZIONE & $\begin{array}{l}\text { 1. RETRASO } \\
\text { 2. DECELERACIÓN }\end{array}$ \\
\hline 145. ROTTAMAZIONE & DESGUACE \\
\hline 146. SCARICAZIONE & DESCARGA \\
\hline 147. SCARNIFICAZIONE & DESCARNADURA \\
\hline 148. SCINTILLAZIONE & 1. - 3. CENTELLEO \\
\hline 149. SCOMUNICAZIONE & EXCOMUNIÓN \\
\hline 150. SCRITTURAZIONE & $\begin{array}{l}\text { 1. ESCRITURACIÓN } \\
\text { 2. CONTRATA } \\
\text { 3. ENCARGO }\end{array}$ \\
\hline 151. SEGRETAZIONE & SIGILO \\
\hline 152. SETTIMANALIZZAZIONE & PUBLICACIÓN SEMANAL \\
\hline 153. SISTEMAZIONE & $\begin{array}{l}\text { 1. DISTRIBUCIÓN } \\
\text { 2. MÉTODO } \\
\text { 3. ARREGLO } \\
\text { 4. ALOJAMIENTO } \\
\text { 5. COLOCACIÓN }\end{array}$ \\
\hline 154. SOGGEZIONE & $\begin{array}{l}\text { 1. SUJECIÓN } \\
\text { 2. RETRAIMIENTO }\end{array}$ \\
\hline 155. SOLLECITAZIONE & $\begin{array}{l}\text { 1. APREMIO } \\
\text { 2. ESFUERZO }\end{array}$ \\
\hline 156. SOMMINISTRAZIONE & 1. e 2. SUMINISTRO \\
\hline 157. SOPPORTAZIONE & $\begin{array}{l}\text { 1. AGUANTE } \\
\text { 2. TOLERANCIA }\end{array}$ \\
\hline 158. SOPRAFFAZIONE & ATROPELLO \\
\hline 159. SOPRASSICURAZIONE & SOBRESEGURO \\
\hline 160. SOSCRIZIONE & 1. e 2. PIE DE IMPRENTA. \\
\hline 161. SOSTRUZIONE & INFRASTRUCTURA \\
\hline 162. SOTTOASSICURAZIONE & SUBSEGURO \\
\hline 163. SOTTOCCUPAZIONE & 1. e 2. SUBEMPLEO \\
\hline 164. SOTTOFONDAZIONE & BASAMENTO \\
\hline
\end{tabular}




\begin{tabular}{|c|c|}
\hline 165. SOVRIMPOSIZIONE & IMPOSICIÓN DE UNA SOBRETASA \\
\hline 166. SPEREQUAZIONE & DESPROPORCIONALIDAD \\
\hline 167. SPORULAZIONE & 1. e 2. ESPOROGÉNESIS \\
\hline 168. STAGNAZIONE & ESTANCAMIENTO \\
\hline 169. STARNUTAZIONE & ESTORNUDO \\
\hline 170. STATUIZIONE & ESTATUTO \\
\hline 171. SUBORNAZIONE & SOBORNO \\
\hline 172. SUFFRAGAZIONE & SUFRAGIO \\
\hline 173. SUGGELLAZIONE & SELLADURA \\
\hline 174. SUPPLICAZIONE & SÚPLICA \\
\hline 175. SVESTIZIONE & DESPOJO \\
\hline 176. TAVOLAZIONE & $\begin{array}{l}\text { 1. EMPADRONAMIENTO } \\
\text { 2. ÁREA }\end{array}$ \\
\hline 177. TERZIARIZZAZIONE & TERCIARIZACIÓN \\
\hline 178. TESAURIZZAZIONE & 1. e 2. ATESORAMIENTO \\
\hline 179. TIPOCOMPOSIZIONE & COMPOSICIÓNTIPOGRÁFICA \\
\hline 180. TITILLAZIONE & COSQUILLEO \\
\hline 181. TRABEAZIONE & ENTABLAMENTO \\
\hline 182. TRACIMAZIONE & DESBORDAMIENTO \\
\hline 183. TRATTAZIONE & TRATADO \\
\hline 184. TRIVELLAZIONE & SONDEO \\
\hline 185. TUMULAZIONE & SEPULTURA \\
\hline 186. TURNAZIONE & ORGANIZACIÓN DEL TRABAJO POR TURNOS \\
\hline 187. UNDAZIONE & DESPLAZAMIENTO DE LAS CAPAS TECTÓNICAS \\
\hline 188. VATICINAZIONE & VATICINIO \\
\hline 189. VENAGIONE & CAZA \\
\hline 190. VESSILLAZIONE & DESTACAMENTO BAJO UN ESTANDARTE \\
\hline 191. VESTIZIONE & $\begin{array}{l}\text { 1. e 2. TOMA DE HÁBITO } \\
\text { 3. INVESTIDURA }\end{array}$ \\
\hline 192. VIDEOCOMUNICAZIONE & VIDEO COMUNICACIÓN \\
\hline 193. VIDEOIMPAGINAZIONE & VIDEO PAGINACIÓN \\
\hline 194. VIDEOINFORMAZIONE & VIDEO INFORMACIÓN \\
\hline 195. VIDEOPROIEZIONE & VIDEO PROYECCIÓN \\
\hline
\end{tabular}




\begin{tabular}{|l|l|}
\hline 196. VIDEOREGISTRAZIONE & VIDEO GRABACIÓN \\
\hline 197. VIDEORIPRODUZIONE & VIDEO REPRODUCCIÓN \\
\hline 198. VIDIMAZIONE & REFRENDO \\
\hline 199. VOMIZIONE & VÓMITO \\
\hline
\end{tabular}

Dai dati analizzati emerge che esistono numerosi suffissi alternativi in spagnolo che esprimono la stessa semantica del suffisso -zione italiano. Di seguito proponiamo una tabella con tutti i casi, il numero di occorrenze e gli esempi presenti nel corpus analizzato.

\begin{tabular}{|c|c|c|}
\hline \multicolumn{3}{|c|}{ SUFFISSI DIVERGENTI DA-CIÓN E NUMERO DI OCCORENZE NEGLI ESEMPI ANALIZZATI } \\
\hline & SUFFISSO & OCCORRENZE \\
\hline \multirow[t]{13}{*}{1} & $-\mathrm{A}$ & 14 \\
\hline & ESCUCHA & 1 \\
\hline & CARGA & 2 \\
\hline & PELEA & 1 \\
\hline & CONSULTA & 1 \\
\hline & DUDA & 1 \\
\hline & MEZCLA & 1 \\
\hline & CEBA & 1 \\
\hline & PEQUISA & 1 \\
\hline & RESERVA & 2 \\
\hline & DESCARGA & 1 \\
\hline & DISPUTA & 1 \\
\hline & SÚPLICA & 1 \\
\hline \multirow[t]{2}{*}{2} & $-\mathrm{ADA}$ & 1 \\
\hline & ESTACADA & 1 \\
\hline \multirow[t]{19}{*}{3} & $-\mathrm{ADO}$ & 21 \\
\hline & ALTERCADO & 2 \\
\hline & EMBETUNADO & 1 \\
\hline & ABONADO & 1 \\
\hline & CAPITULADO & 2 \\
\hline & TALADO & 1 \\
\hline & MECANOGRAFIADO & 1 \\
\hline & DECORADO & 1 \\
\hline & DESACEITADO & 1 \\
\hline & LISTADO & 1 \\
\hline & FORMATEADO & 1 \\
\hline & SULFATADO & 1 \\
\hline & ENTINTADO & 1 \\
\hline & INVENTARIADO & 1 \\
\hline & VALLADO & 1 \\
\hline & ESTAMPADO DE INYECCIÓN & 1 \\
\hline & MAJADO & 2 \\
\hline & ACABADO & 1 \\
\hline & TRATADO & 1 \\
\hline 4 & -ANCIA & 3 \\
\hline
\end{tabular}




\begin{tabular}{|c|c|c|}
\hline & $\begin{array}{l}\text { DISCREPANCIA } \\
\text { LACTANCIA } \\
\text { TOLERANCIA }\end{array}$ & $\begin{array}{l}1 \\
1 \\
1\end{array}$ \\
\hline \multirow[t]{2}{*}{5} & -ANZA & 2 \\
\hline & MATANZA & 2 \\
\hline \multirow[t]{2}{*}{6} & -ARIO & 1 \\
\hline & RECETARIO & 1 \\
\hline \multirow[t]{2}{*}{7} & -ATA & 2 \\
\hline & $\begin{array}{l}\text { SERENATA } \\
\text { CONTRATA }\end{array}$ & $\begin{array}{l}1 \\
1\end{array}$ \\
\hline \multirow[t]{2}{*}{8} & -DURA & 7 \\
\hline & $\begin{array}{l}\text { AÑADIDURA } \\
\text { CABAHIGADURA } \\
\text { DESOLLADURA } \\
\text { MOLEDURA } \\
\text { DESCARNADURA } \\
\text { SELLADURA } \\
\text { INVESTIDURA } \\
\end{array}$ & $\begin{array}{l}1 \\
1 \\
1 \\
1 \\
1 \\
1 \\
1\end{array}$ \\
\hline \multirow[t]{2}{*}{9} & $-E$ & 6 \\
\hline & $\begin{array}{l}\text { DESENGANCHE } \\
\text { DESCIFRE } \\
\text { DEBATE } \\
\text { DESGUACE } \\
\text { AGUANTE } \\
\text { CONTRAINFORME }\end{array}$ & $\begin{array}{l}1 \\
1 \\
1 \\
1 \\
1 \\
1\end{array}$ \\
\hline \multirow[t]{2}{*}{10} & -ENCIA & 8 \\
\hline & $\begin{array}{l}\text { FRECUENCIA } \\
\text { APETENCIA } \\
\text { COMPARECENCIA } \\
\text { CONDESCENDENCIA } \\
\text { TENENCIA } \\
\text { NEGLIGENCIA } \\
\text { CONVERGENCIA }\end{array}$ & $\begin{array}{l}1 \\
1 \\
1 \\
1 \\
2 \\
1 \\
1\end{array}$ \\
\hline \multirow[t]{2}{*}{11} & $-\mathrm{EO}$ & 7 \\
\hline & $\begin{array}{l}\text { COSQUILLEO } \\
\text { SONDEO } \\
\text { RECREO } \\
\text { PARPADEO } \\
\text { CENTELLEO } \\
\end{array}$ & $\begin{array}{l}1 \\
1 \\
1 \\
1 \\
3\end{array}$ \\
\hline \multirow[t]{2}{*}{12} & -GÉNESIS & 2 \\
\hline & ESPOROGÉNESIS & 2 \\
\hline \multirow[t]{2}{*}{13} & -IDAD & 3 \\
\hline & $\begin{array}{l}\text { HOSTILIDAD } \\
\text { FOTOCONDUCTIVIDAD } \\
\text { DESPROPORCIONALIDAD }\end{array}$ & $\begin{array}{l}1 \\
1 \\
1\end{array}$ \\
\hline \multirow[t]{2}{*}{15} & -ÍO & 2 \\
\hline & $\begin{array}{l}\text { DESVARÍ́O } \\
\text { REGADÍO FERTILIZANTE }\end{array}$ & $\begin{array}{l}1 \\
1\end{array}$ \\
\hline
\end{tabular}




\begin{tabular}{|c|c|c|}
\hline \multirow[t]{2}{*}{16} & $-\mathrm{IO}$ & 1 \\
\hline & VATICINIO & 1 \\
\hline \multirow[t]{2}{*}{17} & -ISMO & 2 \\
\hline & $\begin{array}{l}\text { CRONICISMO } \\
\text { NEOLOGISMO }\end{array}$ & $\begin{array}{l}1 \\
1 \\
\end{array}$ \\
\hline \multirow[t]{2}{*}{18} & LOCUZIONI & 49 \\
\hline & $\begin{array}{l}\text { I numerosi esempi di locuzioni so } \\
\text { vengono ripetuti in questa sezione }\end{array}$ & per ragioni di spazio non \\
\hline \multirow[t]{2}{*}{19} & -LOGO & 1 \\
\hline & EPÍLOGO & 1 \\
\hline \multirow[t]{29}{*}{20} & -MIENTO & 57 \\
\hline & FRANQUEAMIENTO & 4 \\
\hline & SANEAMIENTO & 1 \\
\hline & ENCARCELAMIENTO & 1 \\
\hline & ALQUITRANAMIENTO & 1 \\
\hline & AISLAMIENTO & 1 \\
\hline & RECLUTAMIENTO & 1 \\
\hline & ACORTAMIENTO & 1 \\
\hline & DESCORTEZAMIENTO & 2 \\
\hline & DESPLAZAMIENTO & 3 \\
\hline & ENSANCHAMIENTO & 1 \\
\hline & DOLORIMIENTO & 1 \\
\hline & CUMPLIMIENTO & 1 \\
\hline & EXTRAÑAMIENTO & 2 \\
\hline & AFRANCESAMIENTO & 1 \\
\hline & FUSILAMIENTO & 1 \\
\hline & EMBALSAMAMIENTO & 2 \\
\hline & EMPAREDAMIENTO & 1 \\
\hline & PLANTEAMIENTO & 1 \\
\hline & ASENTAMIENTO & 1 \\
\hline & ENCANTAMIENTO & 1 \\
\hline & ENGROSAMIENTO & 1 \\
\hline & ENCABEZAMIENTO & 2 \\
\hline & ALISTAMIENTO & 1 \\
\hline & ROCIAMIENTO & 1 \\
\hline & MEZCLAMIENTO & 1 \\
\hline & ENNOBLECIMIENTO & 2 \\
\hline & OSCURECIMIENTO & 1 \\
\hline & RECONOCIMIENTO & 4 \\
\hline
\end{tabular}




\begin{tabular}{|c|c|c|}
\hline & SIGILO & 1 \\
\hline & MÉTODO & 1 \\
\hline & ARREGLO & 1 \\
\hline & APREMIO & 1 \\
\hline & ESFUERZO & 1 \\
\hline & SUMINISTRO & 2 \\
\hline & ATROPELLO & 1 \\
\hline & SOBRESEGURO & 1 \\
\hline & SUBSEGURO & 1 \\
\hline & SUBEMPLEO & 2 \\
\hline & SOBORNO & 1 \\
\hline & SUFRAGIO & 1 \\
\hline & DESPOJO & 1 \\
\hline & REFRENDO & 1 \\
\hline & VÓMITO & 1 \\
\hline & ESTORNUDO & 1 \\
\hline & ERUCTO & 1 \\
\hline 23 & -ÓN & 1 \\
\hline & UNIÓN & 1 \\
\hline 24 & -SIÓN & 6 \\
\hline & $\begin{array}{l}\text { EROSIÓN } \\
\text { CESIÓN } \\
\text { DISTENSIÓN } \\
\text { DIFUSIÓN } \\
\text { DISCUSIÓN } \\
\text { RECONVERSIÓN } \\
\text { CONEXIÓN }\end{array}$ & $\begin{array}{l}1 \\
1 \\
1 \\
1 \\
1 \\
1 \\
2\end{array}$ \\
\hline 25 & -TURA & 5 \\
\hline & $\begin{array}{l}\text { ABREVIATURA } \\
\text { JUNTURA } \\
\text { INFRASTRUCTURA } \\
\text { SEPULTURA } \\
\end{array}$ & $\begin{array}{l}2 \\
1 \\
1 \\
1\end{array}$ \\
\hline 26 & -ZOMA & 1 \\
\hline & DIAZOMA & 1 \\
\hline
\end{tabular}

Affinché i dati proposti siano chiari in percentuali, presentiamo un grafico che rende palesi le percentuali di uso dei suffissi alternativi a -zione in spagnolo: 


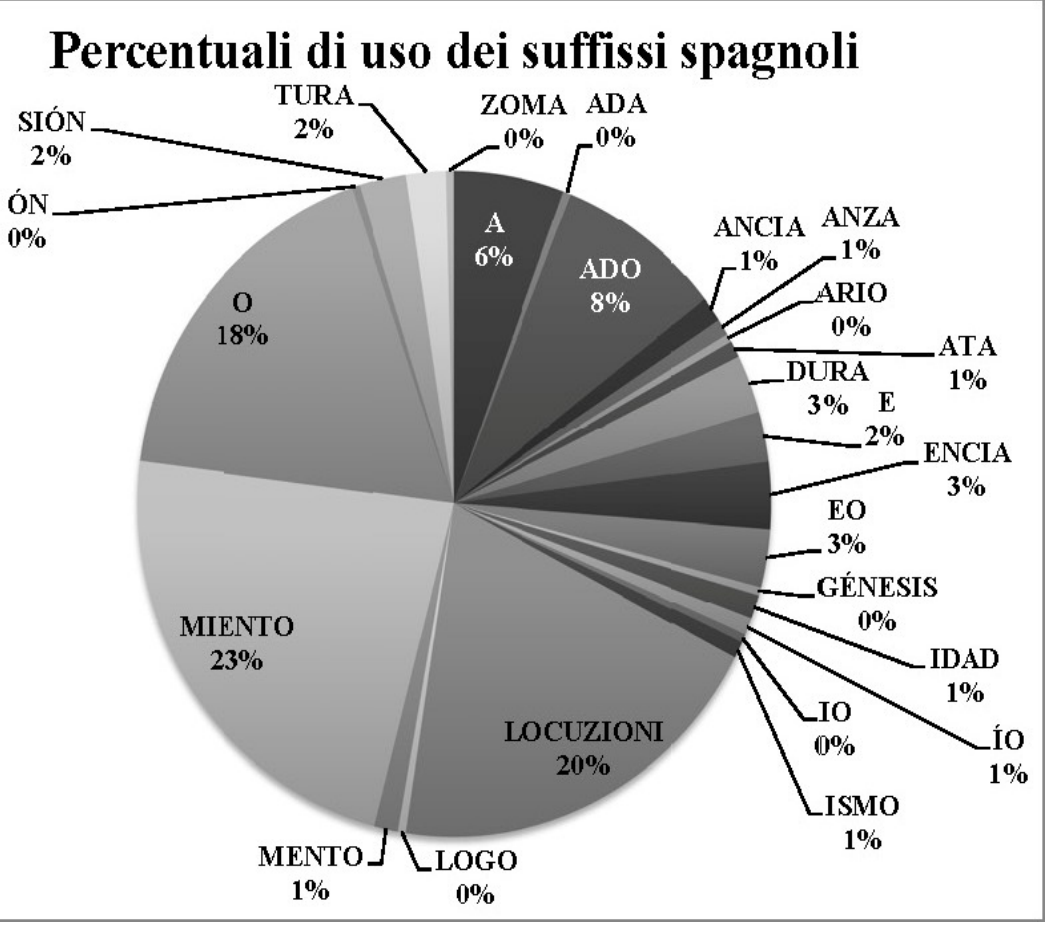

I suffissi più ricorrenti sono: -miento (23\%), le locuzioni (20\%),-o (18\%), -ado (8\%), ed -a (6\%) il resto dei suffissi compare con una percentuale inferiore al $3 \%$ come si può osservare dal grafico e pertanto non significativa, se non addirittura irrilevante.

A questo punto dobbiamo però evidenziare inoltre che nel corpus si presentano diversi casi in cui non solo si produce una divergenza suffissale ma anche una divergenza di base derivativa, come si può osservare in alcuni esempi seguenti:

\begin{tabular}{|l|l|l|}
\hline COIBENTAZIONE & $\begin{array}{l}\text { s. f. [der. di coibentare]. - Loperazione di coibentare e } \\
\text { il risultato; è in genere sinon. Di isolamento nel sign. 3. }\end{array}$ & AISLAMIENTO \\
\hline COLLUTTAZIONE & $\begin{array}{l}\text { s. f. [dal lat. colluctatio -onis]. - Il colluttarsi; rissa, } \\
\text { baruffa: entrare in c., venire a c.; cera stata una furiosa } \\
\text { c.; usci dalla c. con un occhio pesto. In senso fig., vivace } \\
\text { diverbio, scontro polemico. }\end{array}$ & PELEA \\
\hline
\end{tabular}

Questa riflessione ci porta ad ipotizzare che forse il modo di vincolare i dati dei suffissi italiani e spagnoli nel DIES/DEIS dovrebbe cambiare rispetto alla nostra ipotesi iniziale: suffisso italiano = suffisso spagnolo e relativi esempi e considerare que il punto di relazione si possa vincolare al significato dei singoli esempi in ciascuna delle due lingue, instaurando un legame con le basi derivative nonostante tutte le implicazioni che da esso possano derivare. 
4. DISEGNO DELLA BASE DATI E CAMBI DOPO LO STUDIO REALIZZATO SUL SUFFISSO-ZIONE/CIÓN NEL DIES/DEIS.

Di seguito proponiamo una tabella con la struttura della base dati FileMaker Pro che abbiamo disegnato e che esemplifica i cambiamenti che sono stati realizzati rispetto all'idea teorica iniziale e che dovranno essere modificati ulteriormente dopo questo studio dettagliato di tutti gli esempi del suffisso -zione/-ción.

\begin{tabular}{|c|c|}
\hline \multicolumn{2}{|c|}{ Disegno della base dati per il DIES/DEIS/Diseño de base de datos para el DIES/DEIS } \\
\hline NOME DEL PROGETTO & $\begin{array}{l}\text { DISS/DSIS } \\
\text { Dizionario Italiano-Spagnolo di Suffissi/Dizionario } \\
\text { Spagnolo-Italiano di Suffissi }\end{array}$ \\
\hline Nombre DEL PROYECTO & $\begin{array}{l}\text { DIES/DEIS } \\
\text { Diccionario Italiano-Español de Sufijos/ Diccionario } \\
\text { Español-Italiano de Sufijos }\end{array}$ \\
\hline ОвiETTIVO DI QUESTA BASE DATI & Creare un dizionario bilingue di suffissi \\
\hline FinALIDAD DE ESTA BASE DE DATOS & Crear un diccionario bilingüe de sufijos \\
\hline NOME DEL FILE DELLA BASE DATI & DIES_DEIS \\
\hline NOMBRE DE ARCHIVO DE LA BASE DE DATOS & DIES_DEIS \\
\hline NOMBRE DE TABLA I & DizIONARIO ITALIANO DI SUFFISSI \\
\hline NOME DELLA TABELLA 2 & DiCCIONARIO ESPAÑOL DE SUFIJOS \\
\hline NOME DEL TIPO DI CAMPO & Commenti \\
\hline NombRE DE TIPO DE CAMPO & Comentarios \\
\hline LEMA DELLA FORMA BASE & $\begin{array}{l}\text { In questa sezione comparirà il nome del suffisso italiano } \\
\text { e/o spagnolo a seconda del caso }\end{array}$ \\
\hline LEMA FORMA BASE & $\begin{array}{l}\text { En este apartado aparecerá el nombre del sufijo italiano } \\
\text { y/o español según el caso }\end{array}$ \\
\hline \multirow{2}{*}{ - ALLOMORFI/ ALOMORFOS } & $\begin{array}{l}\text { In questa sezione si inseriranno le forme allomorfe dei } \\
\text { suffissi }\end{array}$ \\
\hline & $\begin{array}{l}\text { En este apartado se insertarán las formas alomórficas de } \\
\text { los sufijos }\end{array}$ \\
\hline $\begin{array}{l}\text { RESTRIZIONI/RESTRICCIONES } \\
\text { (CONDICIONES APARICIÓN DEL ALOMORFO) }\end{array}$ & $\begin{array}{ll}\text { - } & \text { Fonetiche/ Fonéticas } \\
\text { - } & \text { Morfologiche/ Morfológicas } \\
\text { - } & \text { Lessicali/Léxicas } \\
\text { - } & \text { Semantiche/Semánticas } \\
\text { - } & \text { Registro/registro }\end{array}$ \\
\hline
\end{tabular}




\begin{tabular}{|c|c|}
\hline \multirow{2}{*}{ DEFINIZIONE/DEFINICIÓN } & $\begin{array}{l}\text { In questa sezione si offriranno dati referenti al tipo } \\
\text { di parole che forma ciascun suffisso e la categoria } \\
\text { grammaticale di procedenza }\end{array}$ \\
\hline & $\begin{array}{l}\text { En este apartado se proporcionarán datos referentes al } \\
\text { tipo de palabras que forma cada uno de los sufijos y la } \\
\text { categoría gramatical de procedencia }\end{array}$ \\
\hline - TIPO DI SUFFISSO & Nominale, aggettivale, verbale \\
\hline - TIPO DE SUFIJO & Nominal, adjetival, verbal \\
\hline $\begin{array}{l}\text { - CATEGORIA GRAMMATICALE DI } \\
\text { PROCEDENZA }\end{array}$ & Deverbale, denominale, deaggettivale \\
\hline $\begin{array}{l}\text { - CATEGORÍA GRAMATICAL DE } \\
\text { PROCEDENCIA }\end{array}$ & Deverbal, denominal, deajetival \\
\hline \multirow{2}{*}{ ETIMOLOGia/ ETIMOLOGÍA } & $\begin{array}{l}\text { In questa sezione apparirà l'informazione etimologica in } \\
\text { modo conciso }\end{array}$ \\
\hline & $\begin{array}{l}\text { En este apartado aparecerá la información etimológica } \\
\text { de forma resumida }\end{array}$ \\
\hline - LINGUA/DIALETTO DI ORIGINE & $\begin{array}{l}\text { Bisognerà decidere se si includono le informazioni } \\
\text { etimologiche sin dall'inizio o se si lasceranno per un } \\
\text { secondo momento }\end{array}$ \\
\hline - LENGUA/Dialecto DE ORIGEN & $\begin{array}{l}\text { Habrá que decidir si se incluyen las informaciones } \\
\text { etimológicas desde el principio o si se dejarán para un } \\
\text { segundo momento }\end{array}$ \\
\hline \multicolumn{2}{|l|}{ - Forma baSE di ORIGINE } \\
\hline \multicolumn{2}{|l|}{ - Forma base de origen } \\
\hline \multicolumn{2}{|l|}{ - Definizione/definición } \\
\hline \multicolumn{2}{|l|}{-TIPO/TIPO } \\
\hline \multicolumn{2}{|l|}{-ORGINE/ORIGEN } \\
\hline \multicolumn{2}{|l|}{ - Cambio di categoria di origine } \\
\hline \multicolumn{2}{|l|}{ - Cambio de categoría origen } \\
\hline \multicolumn{2}{|l|}{ - Denotazione } \\
\hline \multicolumn{2}{|l|}{ • DENOTACIÓN } \\
\hline \multicolumn{2}{|l|}{ - Connotazione } \\
\hline \multicolumn{2}{|l|}{ - conNotación } \\
\hline ESTENSIONE CRONOLOGICA & $\begin{array}{l}\text { In questa sezione comparirà l'epoca in cui il suffisso } \\
\text { comincia ad essere usato o smette di essere usato. }\end{array}$ \\
\hline EXTENSIÓN CRONOLÓGICA & $\begin{array}{l}\text { En este apartado aparecerá la época en la que el sufijo } \\
\text { empieza a ser usado o deja de serlo. }\end{array}$ \\
\hline - Prima testimonianza & $\begin{array}{l}\text { Tutti questi dati cronologici si inseriranno in una fase } \\
\text { successiva }\end{array}$ \\
\hline - Primer testimonio & $\begin{array}{l}\text { Todos estos datos cronológicos se insertarán en una fase } \\
\text { sucesiva }\end{array}$ \\
\hline \multicolumn{2}{|l|}{ - Data o secolo/fecha o siglo } \\
\hline \multicolumn{2}{|l|}{ - EsEMPIO/EJEMPLO } \\
\hline - Secolo di maggior uso i & \\
\hline
\end{tabular}




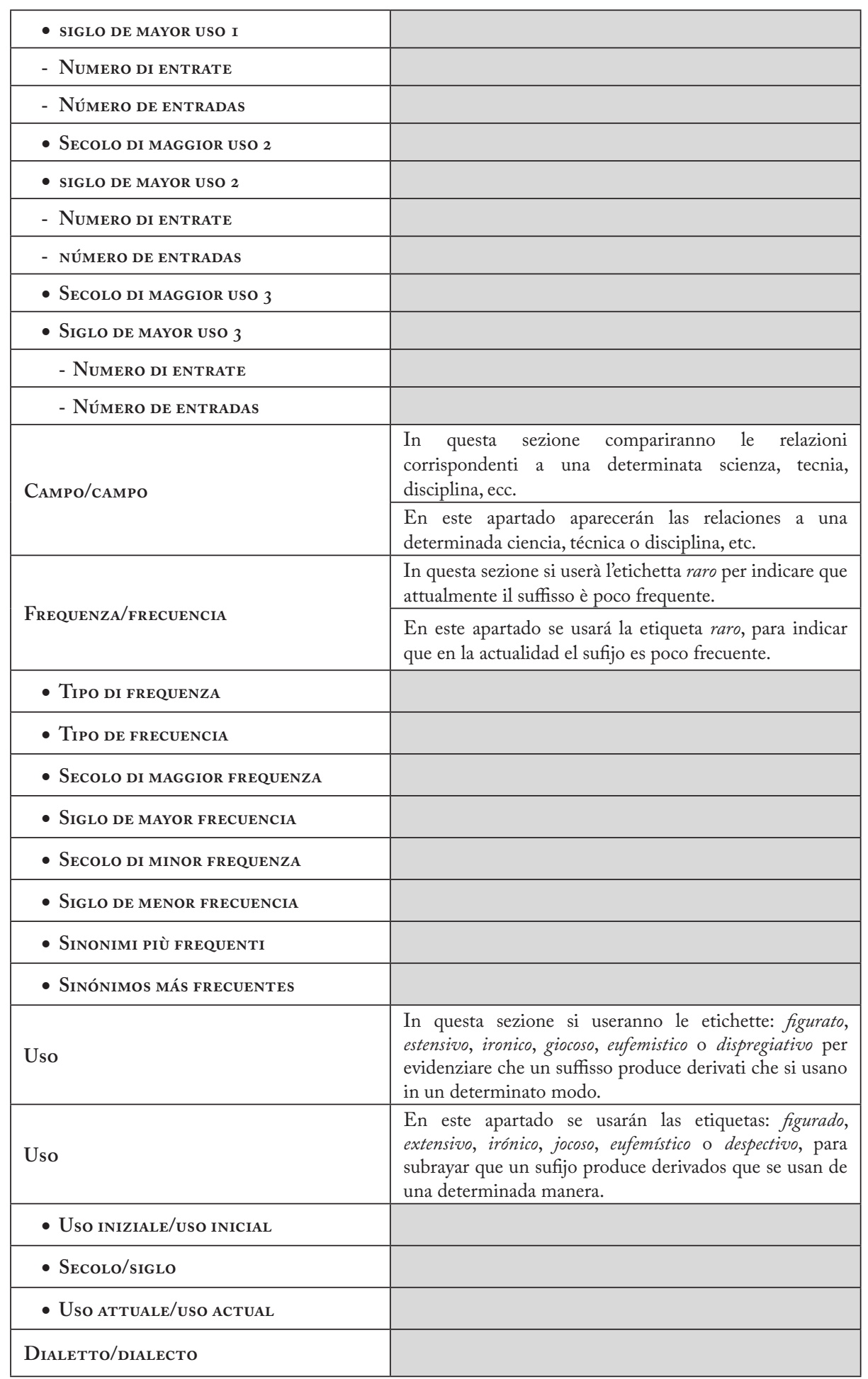




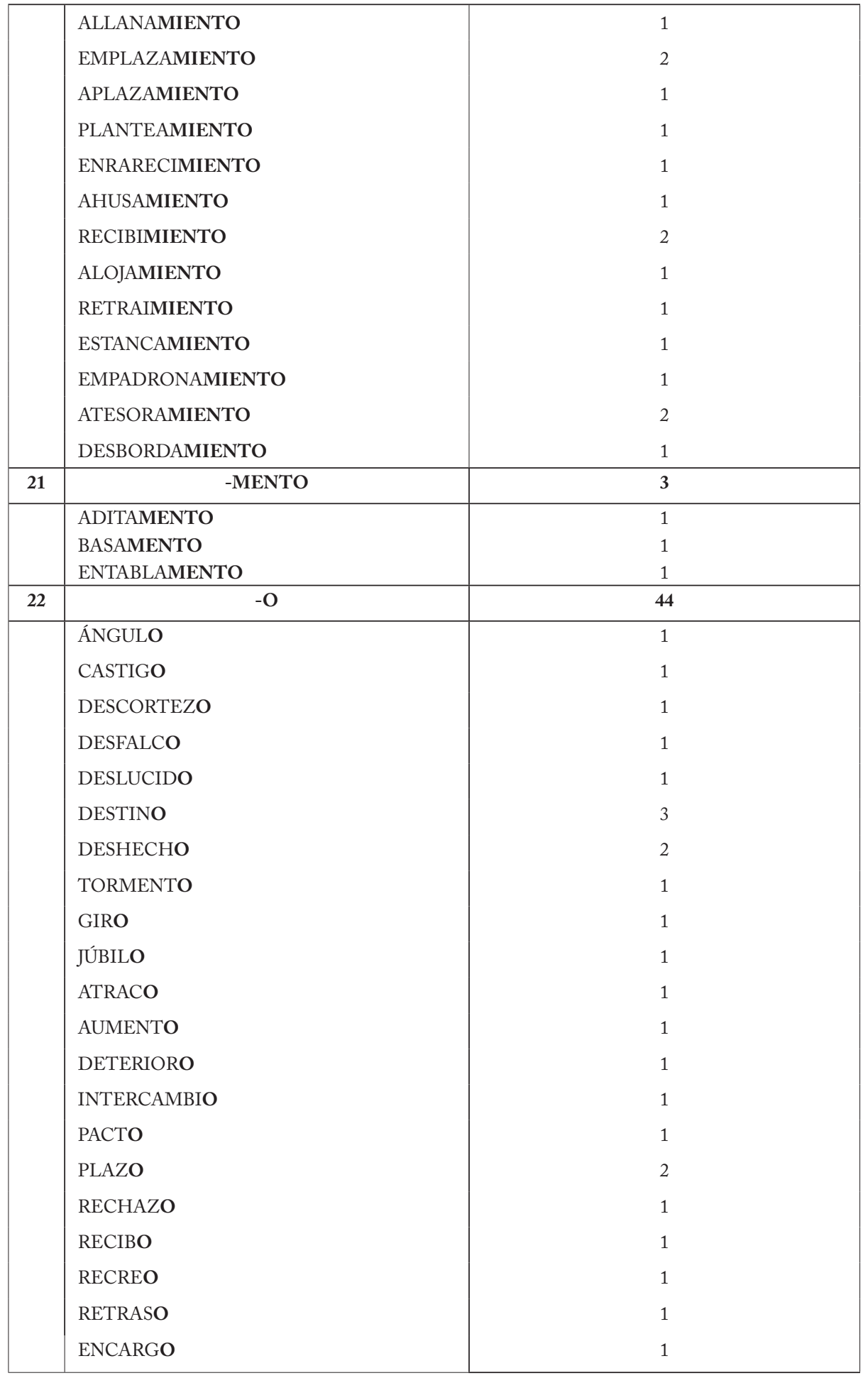




\begin{tabular}{|c|c|}
\hline \multirow{2}{*}{ REgISTRO/REgISTRO } & $\begin{array}{l}\text { Useremo questo campo per marcare un registro familiare, } \\
\text { letterario, popolare, regionale, volgare, ecc. Si indicherà } \\
\text { derivati con queste caratteristiche della stessa famiglia } \\
\text { semantica. }\end{array}$ \\
\hline & $\begin{array}{l}\text { Usaremos este campo para marcar un registro familiar, } \\
\text { literario, popular, regional, vulgar, etc. Se indicará } \\
\text { derivados con estas características de la misma familia } \\
\text { semántica. }\end{array}$ \\
\hline ESEMPI IN SOPPORTO CARTACEO & $\begin{array}{l}\text { Qui si proporranno solo pochi esempi per ovvie ragioni } \\
\text { di spazio }\end{array}$ \\
\hline EJEMPLOS SOPORTE PAPEL & $\begin{array}{l}\text { Aquí se propondrán solo unos pocos ejemplos por } \\
\text { razones obvias de espacio. }\end{array}$ \\
\hline \multicolumn{2}{|l|}{ - FoRMA DERIVATA COMPLETA } \\
\hline \multicolumn{2}{|l|}{ - FoRMa deRIVADA COMPLETA } \\
\hline \multicolumn{2}{|l|}{ - BaSE DERIVATIVA } \\
\hline \multicolumn{2}{|l|}{ - BASE DERIVATIVA } \\
\hline \multicolumn{2}{|l|}{ - Suffisso } \\
\hline \multicolumn{2}{|l|}{ - Sufijo } \\
\hline - Restrizioni & $\begin{array}{l}\text { Bisognerà trovare il modo di giustificare perché quando } \\
\text { esiste un sinonimo in una delle due lingue che copre } \\
\text { la stessa semantica suffissale, a volte (quasi sempre), si } \\
\text { mantiene solo il suffisso e si cambia la base derivativa o si } \\
\text { cambia sia la base derivativa che il suffisso mantenendo } \\
\text { la stessa semantica. }\end{array}$ \\
\hline - Restricciones & $\begin{array}{l}\text { Habría que encontrar la manera de justificar porque } \\
\text { cuando existe un sinónimo en una de las dos lenguas que } \\
\text { cubre la misma semántica sufijal, a veces (casi siempre), } \\
\text { solo se mantiene el sufijo y se cambia la base derivativa o } \\
\text { se cambia base derivativa e incluso el sufijo manteniendo } \\
\text { la misma semántica. }\end{array}$ \\
\hline ESEMPI IN SOPPORTO ELETTRONICO & Tutti gli esempi disponibili \\
\hline EJEMPLOS SOPORTE ELECTRÓNICO & Todos los ejemplos disponibles \\
\hline \multicolumn{2}{|l|}{ - Forma derivata completa } \\
\hline \multicolumn{2}{|l|}{ - Forma derivada completa } \\
\hline \multicolumn{2}{|l|}{ - BASE DERIVATIVA } \\
\hline \multicolumn{2}{|l|}{ - BASE DERIVATIVA } \\
\hline \multicolumn{2}{|l|}{ - Suffisso } \\
\hline \multicolumn{2}{|l|}{ - Sufijo } \\
\hline - Restrizioni & $\begin{array}{l}\text { Bisognerà trovare il modo di giustificare perché quando } \\
\text { esiste un sinonimo in una delle due lingue che copre } \\
\text { la stesa semantica suffissale, a volte (quasi sempre), si } \\
\text { mantiene solo il suffisso e si cambia la base derivativa o si } \\
\text { cambia sia la base derivativa che il suffisso mantenendo } \\
\text { la stessa semantica. }\end{array}$ \\
\hline
\end{tabular}




\begin{tabular}{|c|c|c|c|c|c|c|c|c|c|}
\hline - RESTRICCIONES & \multicolumn{9}{|c|}{$\begin{array}{l}\text { Habría que encontrar la manera de justificar porque } \\
\text { cuando existe un sinónimo en una de las dos lenguas que } \\
\text { cubre la misma semántica sufijal, a veces (casi siempre), } \\
\text { solo se mantiene el sufijo y se cambia la base derivativa } \\
\text { o se cambia base derivativa y hasta sufijo con la misma } \\
\text { semántica }\end{array}$} \\
\hline Transcategorizzazione & \multicolumn{9}{|c|}{$\begin{array}{l}\text { Bisognerà trovare il modo di presentare questo cambio di } \\
\text { categoría, un'idea potrebbe essere quella che propongono } \\
\text { i ricercatori del Guppo di Ricerca: Estructuras de Datos } \\
\text { y Lingüística Computacional dell'Università di Gran } \\
\text { Canaria: }\end{array}$} \\
\hline \multirow[t]{5}{*}{ TRANSCATEGORIZACIÓN } & \multicolumn{9}{|c|}{$\begin{array}{l}\text { Habría que encontrar la forma de presentar este cambio } \\
\text { de categoría, una idea podría ser la que proponen los } \\
\text { investigadores Grupo de Investigación: Estructuras de } \\
\text { Datos y Lingüística Computacional de la Universidad de } \\
\text { Gran Canaria: }\end{array}$} \\
\hline & \begin{tabular}{|l} 
Italiano o \\
Español \\
\end{tabular} & sustantivo & $\%$ & adjetivo & $\%$ & verbo & $\%$ & Total & \\
\hline & \begin{tabular}{|l|} 
sustantivo \\
\end{tabular} & & & & & & & & \\
\hline & \begin{tabular}{|l|} 
adjetivo \\
\end{tabular} & & & & & & & & \\
\hline & \begin{tabular}{|l} 
verbo \\
Total
\end{tabular} & & & & & & & & \\
\hline Grafico statistica d'uso & \multicolumn{9}{|c|}{$\begin{array}{l}\text { Si inserirà qui un grafico per evidenziare la frequenza di } \\
\text { uso dei suffissi a livello diacronico (dalla sua apparizione } \\
\text { fino all'attualità). }\end{array}$} \\
\hline GRÁFICO ESTADÍSTICA DE USO & \multicolumn{9}{|c|}{$\begin{array}{l}\text { Se insertará aquí un gráfico para evidenciar la frecuencia } \\
\text { de uso de los sufijos a nivel diacrónico (desde su aparición } \\
\text { hasta la actualidad). }\end{array}$} \\
\hline
\end{tabular}

Dopo aver realizzato questa ricerca sul suffisso italiano -zione ed il suo equivalente spagnolo -ción, abbiamo potuto constatare che i dati reali presentati dagli esempi suffissati devono essere adattati al modello così come inizialmente lo avevamo concepito. Si rende necessaria una completa ristrutturazione del disegno con la scissione di tutte le parti in tabelle indipendenti ma relazionali, che permettano di attingere ai dati in modo automatico, come nel caso dell'estensione cronologia, gli usi, i campi, registri, ecc. Di tutti gli esempi si dovrà contemplare la possibilità di includere un campo-significato dato che non sempre i vari significati ottengono la stessa traduzione in spagnolo e procedono anche da basi derivazionali diverse, ma questo comporterà dei problemi di gestioni delle informazioni provenienti da tutti gli esempi, poiché spesso pur esistendo un significato che in una delle due lingue viene coperto da una parola diversa, ciò non significa che in una delle due lingue non esiste poi un equivalente esatto del traducente. Come nel caso di:

\begin{tabular}{|l|l|l|}
\hline ALTERAZIONE & $\begin{array}{l}\text { 4. chim. Modificazione delle caratteristiche di } \\
\text { una roccia a causa di agenti esterni o endogeni: a. } \\
\text { delle rocce }\end{array}$ & 4. EROSIÓN \\
\hline
\end{tabular}

Erosione esiste anche in italiano, ma in questo contesto specifico in spagnolo non si contempla l'uso di alteración nel significato specifico punto 4 . 


\section{CONCLUSIONI}

Come affermato in un lavoro precedente (d'Angelis 2009:199-200), le ipotesi realizzate sui corpus di suffissi italiani con corrispondenti spagnoli delle Fasi I e II dei nostri lavori, continuano, anche nel caso specifico del singolo suffisso -zione/-ción, a confermare che lo spagnolo di solito ricorre ad un numero maggiore di suffissi con una stessa semantica per coprire la semantica del suffisso italiano equivalente.

Per poter includere tutti i miglioramenti scaturiti dall'analisi dei singoli esempi del suffisso -zione/-ción presentiamo di seguito una tabella riassuntiva con tutte le tabelle che a nostro avviso dovremmo includere o modificare nel disegno della base dati del DIES/DEIS.

\begin{tabular}{|c|c|c|c|}
\hline $\begin{array}{l}\text { TABELLE DEL } \\
\text { DIZIONARIO }\end{array}$ & CAmpi & $\begin{array}{l}\text { TABELLA CON CUI SI } \\
\text { RELAZIONA IL CAMPO }\end{array}$ & Tipo di RELAZIONE \\
\hline \multirow[t]{9}{*}{$\begin{array}{c}\text { Tabella ia } \\
\text { (SufFissi ITALIANI) }\end{array}$} & $\begin{array}{l}\text { FORMA BASE DEL } \\
\text { SUFFISSO }\end{array}$ & & \\
\hline & Allomorfi & & \\
\hline & $\begin{array}{l}\text { Etimologia deL } \\
\text { SUFFISSO }\end{array}$ & $\begin{array}{c}\text { TABElla } 4 \\
\text { (Etimologia DEL } \\
\text { SufFisso) }\end{array}$ & I A MOLTI \\
\hline & $\begin{array}{l}\text { LiNGUA DI ORIGINE DEL } \\
\text { SUFFISSO }\end{array}$ & Tabella 7 & I A MOLTI \\
\hline & Tipo DI suffisso & $\begin{array}{c}\text { TABElla } 5 \text { (TIPO DE } \\
\text { SUfFISSO) }\end{array}$ & I A MOLTI \\
\hline & $\begin{array}{c}\text { CATEgoria } \\
\text { GRAMMATICALE DI } \\
\text { PROVENIENZA }\end{array}$ & $\begin{array}{c}\text { TABELla } 6 \text { (CATEgoria } \\
\text { GRAMMATICALE DI } \\
\text { PROVENIENZA) }\end{array}$ & I A MOLTI \\
\hline & BASE DERIVATIVA & TABELla 2A & Molti a molti \\
\hline & $\begin{array}{c}\text { Etimologia DELLA } \\
\text { BASE }\end{array}$ & Tabella 3 & Molti a molti \\
\hline & $\begin{array}{l}\text { LiNGUA DI ORIGINE } \\
\text { DELLA BASE }\end{array}$ & Tabella 7 & I A MOLTI \\
\hline \multirow[t]{6}{*}{$\begin{array}{c}\text { TABELLA I B } \\
\text { (suFFISSI SPAGNOLI) }\end{array}$} & $\begin{array}{l}\text { FORMA BASE DEL } \\
\text { SUFISSO }\end{array}$ & & \\
\hline & Allomorfi & & \\
\hline & $\begin{array}{l}\text { Etimologia del } \\
\text { SufFisso }\end{array}$ & $\begin{array}{c}\text { TABElla } 4 \\
\text { (Etimologia DEL } \\
\text { SUfFisso) }\end{array}$ & I A MOLTI \\
\hline & $\begin{array}{l}\text { LINGUA DI ORIGINE DEL } \\
\text { SUFFISSO }\end{array}$ & Tabella 7 & I A MOLTI \\
\hline & TiPo DI suffisso & $\begin{array}{c}\text { TABElla } 5 \text { (TIPO DI } \\
\text { SUfISSO) }\end{array}$ & I A MOLTI \\
\hline & $\begin{array}{c}\text { Categoria } \\
\text { GRAMMATICALE DI } \\
\text { PROVENIENZA }\end{array}$ & $\begin{array}{c}\text { TABELla } 6 \text { (CATEGORIA } \\
\text { GRAMMATICALE DI } \\
\text { PROVENIENZA) }\end{array}$ & I A MOLTI \\
\hline
\end{tabular}




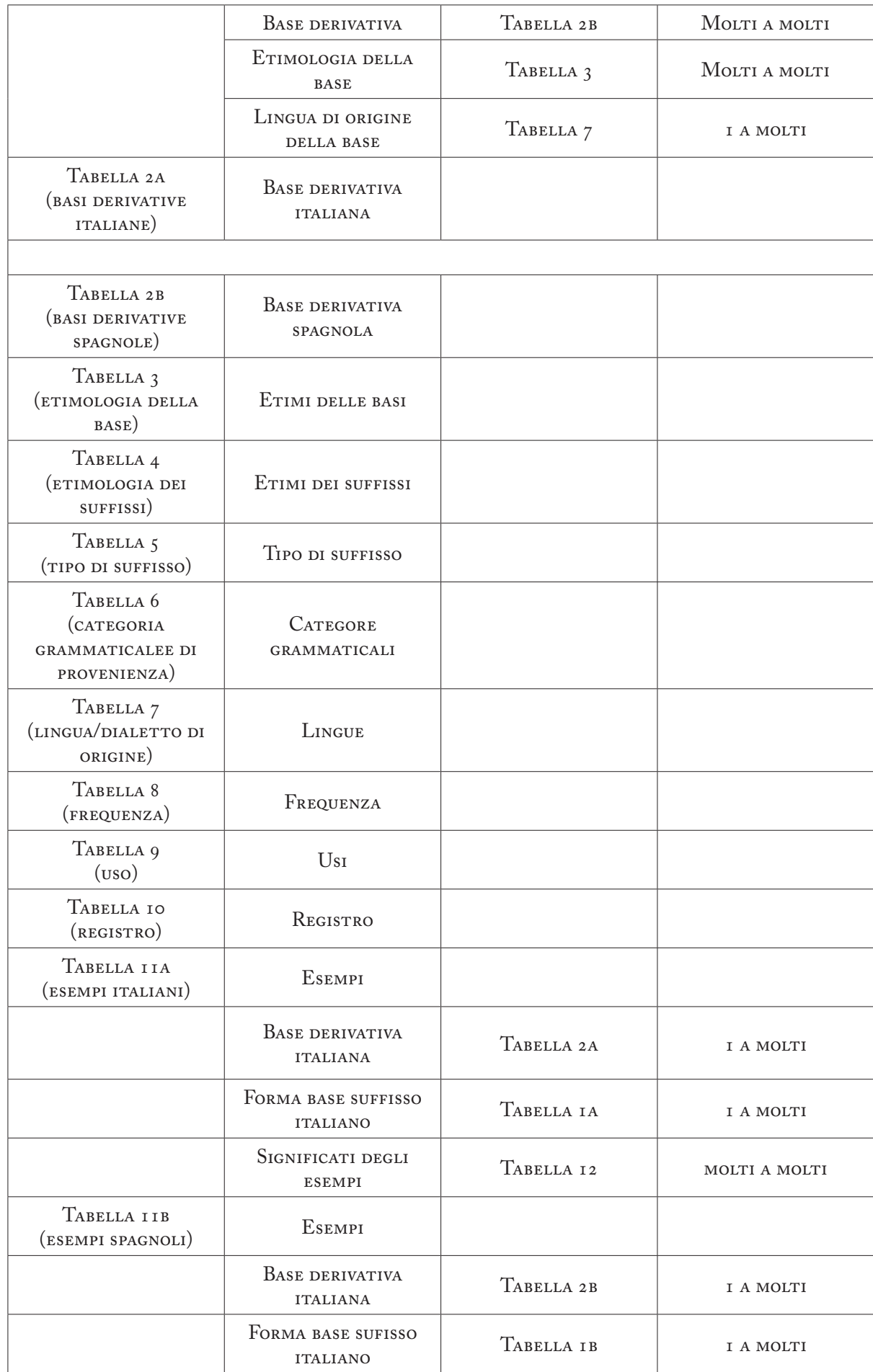




\begin{tabular}{|c|c|c|c|}
\hline & $\begin{array}{c}\text { SigNifiCATI DEGLI } \\
\text { ESEMPI }\end{array}$ & TABella i 2 & MOLTI A MOLTI \\
\hline $\begin{array}{c}\text { TABELLA I } 2 \\
\text { (SIGNIFICATI ITALIANI E } \\
\text { SPAGNOLI) }\end{array}$ & $\begin{array}{l}\text { SignificATI ITALIANI E } \\
\text { SPAGNOLI }\end{array}$ & & \\
\hline
\end{tabular}

\section{BIBLIOGRAFIA}

Battisti, C. - Alessio, G, Dizionario Etimologico Italiano, Firenze, Barbera Editore, Università degli studi di Firenze, 1975.

Calvo Rigual, C. - Giordano, A., Diccionario Italiano-Spagnolo/Spagnolo-Italiano, Barcellona, Herder, 1995.

Corominas, J. - Pascual, J.A., Diccionario crítico etimológico castellano e hispánico, Madrid, Gredos, 1980-1991.

Cortelazzo, M. - Zolli, P., DELI-Dizionario Etimologico della Lingua Italiana con cd-rom, Bologna, Zanichelli, 1999.

D’ANgelis, A. "La derivación nominal y adjetival en italiano y en español: la sufijación", in Actes del VII Congrés de Lingüistica General, Universitat de Barcelona, 2006a, pp. $1-20$.

D'Angelis, A., La derivazione nominale e aggettivale in italiano ed in spagnolo. La suffissazione, Roma, Aracne Editrice, 2006b.

D'ANgelis, A., "La suffissazione nominale in italiano ed in spagnolo", en A. d'Angelis - L. Toppino (eds.), Tendenze attuali nella lingua e nella linguistica italiana in Europa, Roma, Aracne Editrice, (2007), pp. 79-128.

D'Angelis, A., Ancora sulla suffissazione, Roma, Aracne Editrice, 2009.

D'Angelis, A., "DIES/DEIS Diccionario italiano-español de sufijos/Diccionario español-italiano de sufijos: Propuesta inicial de desarrollo", en Lingüistica XL. El lingüista del siglo XXI, Sociedad Española de Lingüística, Madrid, (2012), pp. 27-34.

D’Angelis, A., "Formación y usos de los diminutivos italianos -etto, -ino, -uccio y sus equivalentes españoles", en E. Casanova Herrero - C. Calvo Rigual, (a cura di), Actas del XXVI Congrés Internacional de Lingüistica i Filologia Romàniques, Berlino, Walter de Gruyter GmbH \& Co. KG., (2013), Vol. III, pp. 539-550.

D’Angelis, A. - Núñez Román, F., "DIES/DEIS. Diccionario italiano-español de sufijos/Diccionario español-italiano de sufijos", en Formación de palabras y diacronía, Revista de Lexicografía, (2013), 19, Anexos Universidade da Coruña, pp. 199-215.

Davies, M., Corpus del Español, 100 milioni di parole, 1200s-1900s. Disponible online http://www.corpusdelespanol.org, (2002-).

Dardano, M., La formazione delle parole nell'italiano di oggi, Roma, Bulzoni Editore, 1978.

Dardano, M., Costruire parole: la morfologia derivativa dell'italiano, Bologna, Il Mulino, 2009.

De Mauro, T. (dir.), Grande dizionario italiano dell'uso, con cd-rom, Torino, UTET, 2000-2003. 
Devoto G. - Oli, G.C., Il Dizionario della lingua italiana, con cd-rom, Firenze, Le Monnier, 2002.

DRAE-Diccionario de la Real Academia Española, cd-rom, versione 1.0, Madrid, Espasa Calpe, 2003.

Merz, G., (on line), Dizionario inverso dell'Italiano Moderno: http://culturitalia.uibk. ac.at/wb/WB_it.asp

Pharies, D., Diccionario Etimológico de los sufjos españoles, Madrid, Gredos, 2002.

Picchi, E., (on line), Pitagger: http://serverdbt.ilc.cnr.it/pitagger/default3.htm

Grossmann, M.- Rainer, F. (a cura di), La Formazione delle parole in italiano, Tubinga, Niemeyer, 2004.

Sabatini F. - Coletti, V., DISC-Dizionario Italiano Sabatini Coletti con cd-rom, Firenze, Giunti, 1997.

Sabatini F. - Coletti, V., Il Sabatini Coletti: dizionario della lingua italiana con cdrom, Milano, Sansoni/RCS Libri, 2008.

SÁnchez, A., Gran diccionario de Uso del Español actual, basado en el corpus lingüístico CUMBRE, Madrid. SGEL, 2006.

Santana Suárez, O. - Carreras Riudavets, F. - Pérez Aguiar, J. R., Relaciones morfoléxicas sufjales para el procesamiento del lenguaje natural, Madrid, Editorial Mileto, 2004.

Santana, O. - Pérez, J.R - Carreras, F. J. - Duque, J. d. D. - Hernández, Z. Rodríguez, G., "FLANOM: Flexionador y lematizador automático de formas nominales", Lingüistica Española Actual XXI, 2. Ed. Arco/Libros SL., 1999, pp. 253-297.

Stoppelli, P. - Picchi, E. (a cura di), LIZ 4.0, LITeratura italiana Zanichelli, Bologna, Zanichelli, 2001.

Zanchetta, E. - Baroni, M., "Morph-it! A free corpus-based morphological resource for the Italian language", Proceedings of Corpus Linguistics 2005, Birmingham, University of Birmingham, 2005.

Zingarelli, Lo Zingarelli 2001 (Vocabolario della lingua Italiana) con cd-rom, Bologna, Zanichelli, 2000. 